$$
C_{r s t}=(-2)^{r+s+t} \sum_{n} 2^{-2 n} \frac{n !}{(n-r) !(n-s) !(n-t) !(r+s+t-2 n) !},
$$

where in the summation all factorials have non-negative arguments.

If we transpose the right hand side of (5) and equate coefficients of $u_{1}{ }^{r} u_{2}^{8} u_{3}{ }^{t}$, we have

$$
C_{r s t}=C_{r, s-1, t-1}+C_{r-1, s, t-1}+C_{r-1, s-1, t}+2 C_{r-1, s-1, t-1},
$$

which is the recurrence formula with simplest coefficients, especially as the right hand side can be treated as the sum of five terms. Even though all suffixes vary, it probably provides the quickest way of computing all values of $C_{r s t}$ up to a given set of $r, s, t$. For some machines at least, it may well give the quickest way for calculating a given $C_{r s t}$, and it provides an easy method for desk-machine computation when $r, s$ and $t$ are small. For computations by other methods it provides a simple check. Other checks may be obtained by giving $u_{1}$ special values in (5) and equating coefficients of $u_{2}{ }^{8} u_{3}{ }^{t}$. Putting $u_{1}=-1$, we get

$$
\sum_{r} C_{r s t}=1
$$

given by Gillis and Weiss; putting $u_{1}=-\frac{1}{2}$, we get

$$
\sum_{r} 2^{s+t-r} C_{r s t}=\left(\begin{array}{c}
s+t \\
s
\end{array}\right) \text {. }
$$

Mathematics Department,

Royal College of Science and Technology,

Glasgow, Scotland

1. J. Gillis \& G. Weiss, "Products of Laguerre polynomials," Math. Comp. (MTAC), v. 14,1960 , p. $60-63$.

2. R. D. LoRD, "Some integrals involving Hermite polynomials," London Math. Soc., Jn., v. 24, 1949, p. 101-112.

3. G. N. WATson, "A note on the polynomials of Hermite and Laguerre," London Math. Soc., Jn., v. 13, 1938, p. 29.

\title{
The Evaluation of Certain Probability Integrals
}

\section{By Irwin Greenberg}

A problem which often arises in the field of probability and statistics is the following:

Assume that there are $n$ independent stochastic processes and that the $k$ th process has an output distribution $f_{k}\left(x_{k}\right)$. The probability that the $j$ th process yields a higher output than any of the others is

$$
P_{j}=\int_{-\infty}^{\infty} f_{j}\left(x_{j}\right) \prod_{\substack{k=1 \\ k \neq j}}^{n} \int_{-\infty}^{x_{j}} f_{k}\left(x_{k}\right) d x_{k} d x_{j} .
$$

In certain special cases, equation (1) is easily integrated; for example, if the various $f_{k}\left(x_{k}\right)$ are all uniform or exponential distributions.

Received April 5, 1960; revised May 25, 1960. 
In certain other cases, equation (1) can be put into a form which is readily evaluated by hand computation, using well known statistical reference tables. This paper will illustrate one of these cases; for $n=2$ or 3 and the $x_{k}$ having a normal distribution.

Under the assumption of normality,

$$
f_{k}\left(x_{k}\right)=\left(\sqrt{2 \pi} \sigma_{k}\right)^{-1} \exp \left[-\frac{1}{2}\left(\frac{x_{k}-\mu_{k}}{\sigma_{k}}\right)^{2}\right] ; \quad k=1,2,3
$$

where $\mu_{k}$ and $\sigma_{k}$ are the mean and standard deviation, respectively. Letting

$$
X_{k}=\frac{x_{k}-\mu_{1}}{\sigma_{1}}
$$

equation (2) becomes

$$
f_{k}\left(X_{k}\right)=\left(\sqrt{2 \pi} \sigma_{k}{ }^{\prime}\right)^{-1} \exp \left[-\frac{1}{2}\left(\frac{X_{k}-\mu_{k}{ }^{\prime}}{\sigma_{k}{ }^{\prime}}\right)^{2}\right]
$$

where

$$
\mu_{k}^{\prime}=\frac{\mu_{k}-\mu_{1}}{\sigma_{1}} ; \quad \sigma_{k}{ }^{\prime}=\frac{\sigma_{k}}{\sigma_{1}} .
$$

The probability that the output of process 1 exceeds the output of process 2 is

$$
\begin{aligned}
P_{1} & =\left(2 \pi \sigma_{2}{ }^{\prime}\right)^{-1} \int_{-\infty}^{\infty} \int_{-\infty}^{X_{1}} \exp \left[-\frac{1}{2} X_{1}^{2}-\frac{1}{2}\left(\frac{X_{2}-\mu_{2}{ }^{\prime}}{\sigma_{2}^{\prime}}\right)^{2}\right] d X_{2} d X_{1} \\
& =(\sqrt{2 \pi})^{-1} \int_{-\infty}^{M_{2}} \exp \left(-\frac{t^{2}}{2}\right) d t
\end{aligned}
$$

where

$$
M_{2}=-\mu_{2}^{\prime} / \sqrt{\left(\sigma_{2}^{\prime}\right)^{2}+1} .
$$

The simplification of equation (6) is obtained by expanding the exponent, grouping terms, completing the square in $X_{1}$ and reversing the order of integration after making the transformations:

$$
X_{1}=X_{1}^{\prime} ; X_{2}=X_{1}^{\prime}+X_{2}^{\prime}+\mu_{2}^{\prime} .
$$

Equation (6) is the cumulative normal function and is tabulated in most texts on statistics. In a similar manner, it can be shown that the probability that the output of process 1 exceeds the output of process 2 and of process 3 is

$$
\begin{array}{r}
P_{1}=\left[(2 \pi)^{3 / 2} \sigma_{2}^{\prime} \sigma_{3}{ }^{\prime}\right]^{-1} \int_{-\infty}^{\infty} \int_{-\infty}^{X_{1}} \int_{-\infty}^{X_{1}} \exp \left[-\frac{1}{2} X_{1}{ }^{2}-\frac{1}{2}\left(\frac{X_{2}-\mu_{2}{ }^{\prime}}{\sigma_{2}{ }^{\prime}}\right)^{2}\right. \\
\left.-\frac{1}{2}\left(\frac{X_{3}-\mu_{3}{ }^{\prime}}{\sigma_{3}{ }^{\prime}}\right)^{2}\right] d X_{3} d X_{2} d X_{1} \\
=\left[2 \pi \sqrt{1-r^{2}}\right]^{-1} \int_{-\infty}^{M_{2}} \int_{-\infty}^{M_{3}} \exp \left[-\frac{1}{2\left(1-r^{2}\right)}\left(t_{2}{ }^{2}-2 r t_{2} t_{3}\right.\right. \\
\left.\left.+t_{3}{ }^{2}\right)\right] d t_{3} d t_{2},
\end{array}
$$


where

$$
\left\{\begin{aligned}
M_{2} & =-\mu_{2}^{\prime} / \sqrt{\left(\sigma_{2}\right)^{2}+1} \\
M_{3} & =-\mu_{3}^{\prime} / \sqrt{\left(\sigma_{3}\right)^{2}+1} \\
\frac{1}{r} & =\sqrt{\left(\sigma_{3}\right)^{2}+1} \sqrt{\left(\sigma_{2}^{\prime}\right)^{2}+1}
\end{aligned}\right.
$$

Equation (10) gives the volume under the bivariate normal probability surface with correlation coefficient $r$. These volumes are tabulated in [1].

AVCO Research and Advanced Development

Wilmington, Massachusetts

1. NBS Applied Mathematics Series, No. 50, Tables of the Bivariate Normal Distribution Function and Related Functions, U. S. Government Printing Office, Washington, D. C. 1959.

\section{The Congruence $2^{p-1} \equiv 1\left(\bmod p^{2}\right)$ for $p<100,000$}

\section{By Sidney Kravitz}

Fröberg has previously announced [1] the computation of the Fermat remainders corresponding to all odd primes less than 50,000. His results show that $p=1093$ and $p=3511$ are the only solutions of the congruence $2^{p-1} \equiv 1\left(\bmod p^{2}\right)$ in that range.

The residues of $2^{p-1}\left(\bmod p^{2}\right)$ have been computed for 50,000 $<p<100,000$ on an IBM 650 system at Picatinny Arsenal. No residue congruent to 1 was found corresponding to a prime in this range.

A copy of the table of residues has been deposited in the Unpublished Mathematical Tables file.

\section{Picatinny Arsenal}

Dover, New Jersey

1. C. E. FröBerg, "Some Computations of Wilson and Fermat Remainders," $M T A C$, v. 12,1958, p. 281.

Editorial Note: Reference should also be made to:

1. W. Meissner, "Uber die Teilbarkeit von $2^{p}-2$ durch das Quadrat der Primzahl $p=$ 1093" Akad. d. Wiss, Berlin, Sitzungsb., v. 35, 1913, p. 663-667

2. N. G. W. H. BEEGER, "On a new case of the congruence $2^{p-1} \equiv 1\left(\bmod p^{2}\right)$, " Messenger Math., v. 51, 1922, p. 149-150.

Received April 14, 1960. 